\title{
Simple method for calculating the speed of sound in tight-binding models: Application to the Su-Schrieffer-Heeger model
}

\author{
Fernando L. J. Vos, Daniel P. Aalberts, and Wim van Saarloos \\ Instituut-Lorentz for Theoretical Physics, Leiden University, P.O. Box 9506, 2300 RA Leiden, The Netherlands
}

(Received 25 September 1995)

\begin{abstract}
We present a simple general method for calculating the speed of sound in conjugated polymers, as modeled by one-dimensional tight-binding Hamiltonians. We show how the elastic constants can easily be calculated by imposing a uniform strain on the system. This in turn yields the speed of sound. To illustrate the method we consider the Su-Schrieffer-Heeger model for polyacetylene. We compare our exact analytical expression for the speed of sound as a function of the electron-phonon coupling strength to results obtained previously by other authors, and we verify our results by means of numerical simulations. Most previous results grossly overestimate the renormalization of the speed of sound.
\end{abstract}

Conjugated polymers such as polyacetylene can be understood with concepts borrowed from the theory of onedimensional metals. For example, the alternation between carbon single and double bonds is a consequence of the fact that dimerizing the lattice lowers the electronic energy at the Fermi level. This is the Peierls instability. Polyacetylene, $(\mathrm{CH})_{x}$, has a $\sigma$-bonded backbone and a valence band comprised of $\pi$ electrons. An important model for this system was proposed by $\mathrm{Su}$, Schrieffer, and Heeger. ${ }^{1-4}$ They model these physical elements in a linear tight-binding Hamiltonian in which the $\pi$ electrons are treated quantum mechanically but in which the atomic coordinates and $\sigma$ bonds are treated classically. This theoretical framework simplifies calculations and exposes the basic physical mechanisms when the molecule is excited from its ground state.

In addition to including dimerization, the extended $\pi$-electron system provides a screening mechanism which reduces the speed of sound. ${ }^{5}$ A simplified calculation of this effect will be the subject of this paper. The sound velocity and the optical frequency have previously been calculated perturbatively using the dimensionless $\pi$-electron-phonon coupling constant $\lambda$ as the small parameter. These calculations lead to erroneous results which subsequently were often quoted in the literature. ${ }^{2,3}$ Here we will present a physically transparent and exact method of calculating the speed of sound and the optical frequency for long wavelengths. The calculation rests on elementary arguments that are borrowed from continuum elastic theory and assumptions on which models like the Su-Schrieffer-Heeger (SSH) model rest. Below we will first give the general idea on which our method is based, after which we will demonstrate the method by applying it to the SSH model. It should be clear, however, that the method is quite general in that it is applicable to a wide class of tight-binding models, both in one and in higher dimensions.

The basic idea underlying our approach is very simple. Consider for example a one-dimensional elastic medium. Long wavelength distortions in such a medium are governed by the continuum Lagrangian

$$
L=\int d x\left[\frac{1}{2} \rho\left(\frac{\partial U}{\partial t}\right)^{2}-\frac{1}{2} C\left(\frac{\partial U}{\partial x}\right)^{2}\right]
$$

Here $U(x, t)$ denotes the displacement of a small volume with mass density $\rho$, away from its position in the homogeneous, undistorted state, and $C$ is an elastic coefficient. In the more general three-dimensional formulation such coefficients are known as Lamé coefficients. ${ }^{6}$ We now apply this elastic theory to a solid with lattice parameter $a$, by applying a uniform stretch $\delta$, so that the lattice parameter $a$ of the medium changes into $a+\delta$. Thus, $C(\partial U / \partial x)^{2}=C(\delta / a)^{2}$. The second term in Eq. (1) then is nothing more than the second-order change in energy density of the medium in the presence of a uniform stretch. In terms of the energy per lattice site $\varepsilon$ we therefore have

$$
C=a \varepsilon_{\delta \delta},
$$

where $\varepsilon_{\delta \delta}$ denotes the second derivative of $\varepsilon$ with respect to a uniform stretch $\delta$. Since the wave equation associated with Eq. (1) is $\rho\left(\partial^{2} U / \partial t^{2}\right)=C\left(\partial^{2} U / \partial x^{2}\right)$, we immediately have for the speed of sound $c$

$$
c=\sqrt{\frac{C}{\rho}}=a \sqrt{\frac{\varepsilon_{\delta \delta}}{M}},
$$

where $M=a \rho$ is the mass per lattice site. That it is not necessary to study finite wave vector $q$ oscillations and then take the limit $q \rightarrow 0$ to calculate the speed of sound, can be understood as follows. Uniform stretch is in fact the $q=0$ mode when positions are expressed in relative coordinates.

Our analysis will be based on the observation that the energy density $\varepsilon$, as a function of the stretch parameter $\delta$, can be calculated nonperturbatively rather easily in onedimensional tight-binding models. Combined with Eq. (2) this immediately gives the sound velocity. The only complication in applying this idea lies in the fact that as a result of the electron-phonon interaction, changes in the stretch $\delta$ are accompanied by changes in the dimerization amplitude $u$. The exact generalization of Eq. (2) to this more general case is given later in this paper [Eq. (17) below], and is based on the fact that the optical frequencies are much higher than the acoustical frequencies for long wavelengths, so that it is permissible to average over the fast optical modes in deriving the effective elastic energy as a function of the stretch $\delta$. We 
note that this simple physical picture does not seem to have been exploited before. For example, previous approaches for the SSH model have usually been based on uncontrolled perturbation expansions. ${ }^{8}$

In this paper, we illustrate our approach by applying it to the SSH model. This enables us to compare our results for the sound velocity to those obtained with different methods. This comparison will disprove the often quoted result, that the suppression of the sound velocity is linear in the electron-phonon coupling constant $\lambda$ for small coupling., ${ }^{2,3}$ Instead we find that this reduction is exponentially small for weak coupling. The validity of our physically transparent and technically efficient approach is further demonstrated, by comparing it with numerical simulations.

The SSH Hamiltonian is given by

$$
H=H_{\mathrm{el}}+H_{l},
$$

with the $\pi$-electron-lattice coupling written as

$$
\begin{aligned}
H_{\mathrm{el}}= & -\sum_{s} \sum_{n=1}^{N-1}\left[t-\alpha\left(u_{n+1}-u_{n}\right)\right] \\
& \times\left[c_{n, s}^{\dagger} c_{n+1, s}+\text { H.c. }\right],
\end{aligned}
$$

and the lattice part

$$
H_{l}=\frac{K}{2} \sum_{n=1}^{N-1}\left(u_{n+1}-u_{n}\right)^{2}+\frac{M}{2} \sum_{n=1}^{N} \dot{u}_{n}^{2} .
$$

In Eqs. (5) and (6), $n$ numbers the $(\mathrm{CH})$ groups 1 through $N, u_{n}$ is the displacement along the chain of the $n$th $(\mathrm{CH})$ group relative to some reference position $n a, c_{n, s}^{\dagger}\left(c_{n, s}\right)$ creates (annihilates) an electron with spin projection $s$ at site $n$. The model parameters are $t$ the hopping parameter for uniform spacing $a$ between adjacent $(\mathrm{CH})$ groups, $\alpha$ the electron-phonon coupling constant, $K$ the force constant for bond length deviations from equal spacing of the $\sigma$-bonding backbone, and $M$ the mass of a $(\mathrm{CH})$ group. The total chain length $N$ will be assumed to be large enough to justify the neglect of end effects.

The $\pi$-electron-lattice part of the Hamiltonian $H_{\mathrm{el}}$ models the coupling of the $\pi$-electrons to the lattice degrees of freedom, via a linear modulation with distance of the hopping frequency $t$. The first term in the lattice part of the Hamiltonian $H_{l}$ models a harmonic restoring force on the $\sigma$-bonded $(\mathrm{CH})$ groups when displaced from equal spacing $a$, and the second term is the kinetic energy.

An expression for the energy of the ground state with uniform dimerization amplitude $u$ is usually obtained by minimization of the energy after substituting the ansatz $u_{n}=(-1)^{n} u$. Here we take a slightly different ansatz to accommodate a uniform bond stretching $\delta$ :

$$
u_{n}=(-1)^{n} u+n \delta
$$

Periodic boundary conditions are incompatible with this ansatz, since $u_{N}=u_{1}$ can only be imposed for $\delta=0$. To circumvent this technical problem, we envisage a long chain with boundary conditions such, that there is no stretching in the ground state. Details on how this can be implemented explic- itly will be presented in a forthcoming paper, ${ }^{7}$ but we stress that the results we derive here are insensitive to boundary conditions.

Substitution of Eq. (7) in Eq. (4) renders the Hamiltonian

$$
\begin{aligned}
H(u, \delta)= & -\sum_{n, s}\left[t+2 \alpha(-1)^{n} u-\alpha \delta\right]\left[c_{n, s}^{\dagger} c_{n+1, s}^{+}+\text {H.c. }\right] \\
& +2 N K u^{2}+\frac{1}{2} N K \delta^{2}
\end{aligned}
$$

The procedure for diagonalizing the Hamiltonian [Eq. (8)] is straightforward and follows the standard procedure discussed elsewhere. $^{2-4,7}$ Here we note that the expression for the ground-state energy-per-site with $\delta \neq 0$ can be obtained from the corresponding expression in the literature ${ }^{2,3}$ for $\delta=0$, if one replaces $t$ by $t-\alpha \delta$ in the latter and adds the elastic term $K \delta^{2} / 2$. The energy-per-site then becomes

$$
\varepsilon(u, \delta)=\frac{-4(t-\alpha \delta)}{\pi} \mathscr{E}\left(\sqrt{1-z^{2}}\right)+2 K u^{2}+\frac{1}{2} K \delta^{2}
$$

where we have introduced the dimensionless variable $z \equiv 2 \alpha u /(t-\alpha \delta)$, and where $\mathscr{E}$ is the complete elliptic function of the second kind.

As explained above, the sound velocity can be expressed simply in terms of the second-derivatives $\varepsilon_{\delta \delta}$, etc., as these play the role of elastic coefficients. Expanding the energyper-site $\varepsilon(u, \delta)$ about the equilibrium values $(\underline{u}, \underline{\delta}=0)$ to second order, we have

$$
\Delta \varepsilon(u, \delta)=\frac{1}{2} \varepsilon_{u u}(\Delta u)^{2}+\varepsilon_{u \delta}(\Delta u)(\Delta \delta)+\frac{1}{2} \varepsilon_{\delta \delta}(\Delta \delta)^{2},
$$

where $\Delta \varepsilon(u, \delta) \equiv \varepsilon(u, \delta)-\varepsilon(\underline{u}, \underline{\delta}), \Delta u \equiv u-\underline{u}$, etc. From Eq. (9) the second derivatives, evaluated at $\delta=0$, are obtained as

$$
\begin{gathered}
\varepsilon_{u u}=K\left[8 \lambda \frac{2 \mathscr{E}-\left(1+z^{2}\right) \mathscr{K}}{\left(1-z^{2}\right)^{2}}+4\right], \\
\varepsilon_{\delta \delta}=K\left[2 \lambda \frac{2 \mathscr{E}-\left(1+z^{2}\right) \mathscr{K}}{\left(1-z^{2}\right)^{2}}+1\right], \\
\varepsilon_{u \delta}=K\left[4 \lambda \frac{2 \mathscr{E}-\left(1+z^{2}\right) \mathscr{K}}{\left(1-z^{2}\right)^{2}}\right],
\end{gathered}
$$

where $\lambda$ is the dimensionless electron-phonon coupling strength defined through $\lambda=2 \alpha^{2} /(\pi K t)$, $\mathscr{K}$ is the complete elliptic function of the first kind, and where we have abbreviated $\mathscr{E}\left(\sqrt{1-z^{2}}\right)$ and $\mathscr{K}\left(\sqrt{1-z^{2}}\right)$ as $\mathscr{E}$ and $\mathscr{K}$.

Due to the coupling between the strain and the dimerization amplitude, through the coefficient $\varepsilon_{u \delta}=\varepsilon_{\delta u}$, the expression [Eq. (3)] for the sound velocity in a simple medium is not appropriate. An explicit derivation of the more general expression [Eq. (17)] below, will be given elsewhere; ${ }^{7}$ here we content ourselves with a simple heuristic argument: due to the arbitrarily large difference in the frequency of acoustic $(\delta)$ versus optical $(u)$ modes in the $q \rightarrow 0$ limit, we may approximate 


$$
\left.\overline{\frac{\partial \varepsilon(u, \delta)}{\partial u}}\right|_{u=\underline{u}}=0,
$$

where the overbar indicates a time average on a time scale short compared to acoustical periods but long compared to optical periods. Put differently, the forces in the optical modes average to zero on acoustic time scales. We can obtain an expression for the value of the time averaged $\overline{\Delta u}$ for a given quasistatic stretch $\Delta \delta$, by expanding Eq. (14):

$$
\varepsilon_{u u} \overline{\Delta u}+\varepsilon_{u \delta} \Delta \delta=0
$$

so that the optical modes fluctuate about $\overline{\Delta u}=-\left(\varepsilon_{u \delta} / \varepsilon_{u u}\right) \Delta \delta$ for a given stretch $\Delta \delta$. On substitution in Eq. (10) we arrive at an expansion for the effective energy with respect to the stretch $\Delta \delta$, valid on acoustic timescales

$$
\Delta \varepsilon=\frac{1}{2}\left(\varepsilon_{\delta \delta}-\frac{\varepsilon_{u \delta}^{2}}{\varepsilon_{u u}}\right)(\Delta \delta)^{2} .
$$

Along the same lines as above, we then obtain for the speed of sound in the dimerized ground state

$$
c=c_{0} \sqrt{\frac{\varepsilon_{\delta \delta}}{K}-\frac{\varepsilon_{u \delta}^{2}}{K \varepsilon_{u u}}},
$$

where $c_{0}=a \sqrt{K / M}$ is the bare speed of sound and where the second derivatives given in Eqs. (11)-(13) are to be evaluated at the equilibrium values $\underline{u}$ and $\underline{\delta}=0$. We stress that, within the adiabatic approximation for the electron dynamics, Eq. (17) is exact. Figure 1(a) shows the reduction of the sound velocity as a function of the coupling strength $\lambda$. Note that, by expanding the coefficients in Eq. (17), we find an exponentially small renormalization of the sound velocity for small $\lambda$ :

$$
c / c_{0} \sim 1-4\left(\frac{1}{\lambda}-2\right) e^{-(2+1 / \lambda)} .
$$

On the one hand, this contradicts the often quoted result ${ }^{2,3}$ that the sound velocity for small coupling is given by $c=c_{0} \sqrt{1-2 \lambda}$, and the dashed line in Fig. 1(a) shows this behavior of the sound velocity as obtained by Rice et $a l .{ }^{8}$ On the other hand, Psaltakis and Papanicolaou ${ }^{9}$ used a $1 / n$ expansion technique for calculating the phonon spectrum, where $n$ is the degeneracy of the $\pi$-electron bands ( $n=2$ for polyacetylene), and their result for the suppression of the sound velocity compares extremely well to our exact result.

To illustrate the validity of Eq. (17), we have also performed numerical simulations in which a sinusoidal modulation was superimposed on the dimerized ground state. The sound velocities were calculated by measuring the resulting periods of oscillations for different $\lambda$. As is clear from Fig. 1(a), where the diamonds represent the simulation results, the agreement with Eq. (17) is excellent.

It is perhaps not surprising that the effect of the coupling on the sound velocity is only exponentially small in the weak-coupling limit, and roughly linear for larger couplings. After all, the effect is a manifestation of the change in chain stiffness which in turn is directly related to the electronic
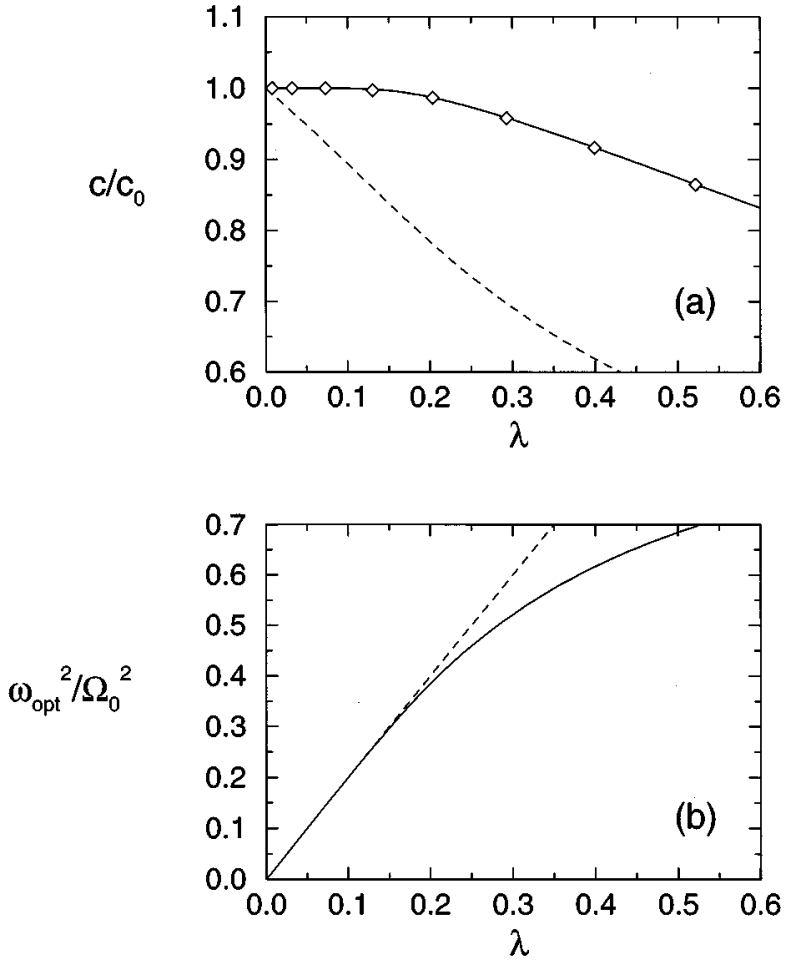

FIG. 1. (a) Suppression of the sound velocity as a function of the coupling strength $\lambda$, defined after Eq. (13). The solid line shows our analytical result [Eq. (17)]. The dashed line shows the result obtained by Rice et al. (Ref. 8). The diamonds mark the points we obtained by a numerical simulation (see text), which unambiguously show the validity of our approach. (b) Long wavelength optical frequency as a function of the coupling strength $\lambda$. The solid line depicts the exact result [Eq. (19)]. The dashed line shows the weak-coupling approximation [Eq. (20)].

gap. It is well known that the gap is exponentially small in weak coupling, crossing over to linear in $\lambda$ behavior for larger couplings.

Since optical frequencies are finite as $q \rightarrow 0$, the optical frequency is given in terms of $\varepsilon_{u u}$ only: ${ }^{7}$

$$
\begin{aligned}
\frac{\omega_{\mathrm{opt}}^{2}}{\Omega_{0}^{2}} & =\frac{\varepsilon_{u u}}{4} \\
& =2 \lambda \frac{2 \mathscr{E}-\left(1+z^{2}\right) \mathscr{R}}{\left(1-z^{2}\right)^{2}}+1,
\end{aligned}
$$

where $\Omega_{0}=\sqrt{4 K / M}$ is the frequency at the band edge at $\pm \pi / a$ in the absence of dimerization. The solid line in Fig. 1(b) shows the behavior of this optical frequency as a function of $\lambda$, while the dashed line depicts the weak-coupling result

$$
\omega_{\mathrm{opt}}=\sqrt{2 \lambda} \Omega_{0},
$$

obtained from Eq. (19). Note that, in contrast to the weakcoupling behavior [Eq. (18)] of the sound velocity, our weakcoupling result for the optical frequency [Eq. (20)] and the result given in the literature ${ }^{2,3,8}$ agree.

The discrepancy between our exact result for the sound velocity and the result ${ }^{8}$ obtained using perturbation theory in 
$\lambda$, can be explained by noting that the weak-coupling behavior [Eq. (18)] shows an essential singularity at $\lambda=0$. This behavior can therefore never be obtained using perturbation theory in $\lambda$. On the other hand, the analytic behavior for small $\lambda$ of the optical frequency [Eq. (20)] can be obtained correctly using perturbation theory.

In summary, we have presented a technically simple and physically transparent way of obtaining expressions for the sound velocity and optical frequency which can easily be applied to other models. These quantities may serve as a guide to determine the validity of spectra calculated by other means.

F.L.J.V. and D.P.A. were supported by Stichting voor Fundamenteel Onderzoek der Materie (FOM), which is financially supported by the Nederlandse Organisatie voor Wetenschappelijk Onderzoek (NWO).
${ }^{1}$ W.P. Su, J.R. Schrieffer, and A.J. Heeger, Phys. Rev. Lett. 42, 1698 (1979).

${ }^{2} \mathrm{Yu}$ Lu, Solitons \& Polarons in Conducting Polymers (World Scientific, Singapore, 1988).

${ }^{3}$ A.J. Heeger, S. Kivelson, J.R. Schrieffer, and W.P Su, Rev. Mod. Phys. 60, 781 (1988).

${ }^{4}$ D. Baeriswyl, in Theoretical Aspects of Band Structures and Electronic Properties of Pseudo-One-Dimensional Solids, edited by H. Kamimura (Reidel, Dordrecht, 1985).
${ }^{5}$ L. Salem, Molecular Orbital Theory of Conjugated Systems (Benjamin, London, 1966).

${ }^{6}$ See, e.g., L.D. Landau and E.M. Lifshitz, Theory of Elasticity (Pergamon, New York, 1986).

${ }^{7}$ F.L.J. Vos, D.P. Aalberts, and W. van Saarloos (unpublished).

${ }^{8}$ M.J. Rice, S.R. Phillpot, A.R. Bishop, and D.K. Campbell, Phys. Rev. B 34, 4139 (1986).

${ }^{9}$ G.C. Psaltakis and N. Papanicolaou, in Interacting Electrons in Reduced Dimensions, edited by D. Baeriswyl and D.K. Campbell (Plenum Press, New York, 1988). 